\title{
Le « Jeu de l'amour » : trajectoires sacrificielles et usages stratégiques des martyrs dans le mouvement sikh pour le Khalistan
}

\section{Laurent Gayer}

\section{(2) OpenEdition Journals}

Édition électronique

URL : http://journals.openedition.org/conflits/2101

DOI : $10.4000 /$ conflits.2101

ISSN : 1777-5345

Éditeur :

CCLS - Centre d'études sur les conflits lilberté et sécurité, L'Harmattan

Édition imprimée

Date de publication : 1 novembre 2006

Pagination : 113-133

ISBN : 2-296-01436-4

ISSN : 1157-996X

Référence électronique

Laurent Gayer, «Le « Jeu de l'amour » : trajectoires sacrificielles et usages stratégiques des martyrs dans le mouvement sikh pour le Khalistan », Cultures \& Conflits [En ligne], 63 | automne 2006, mis en ligne le 09 novembre 2006, consulté le 30 mars 2021. URL : http://journals.openedition.org/conflits/ 2101 ; DOI : https://doi.org/10.4000/conflits.2101

Ce document a été généré automatiquement le 30 mars 2021

Creative Commons License 


\title{
Le « Jeu de l'amour» : trajectoires sacrificielles et usages stratégiques des martyrs dans le mouvement sikh pour le Khalistan
}

\author{
Laurent Gayer
}

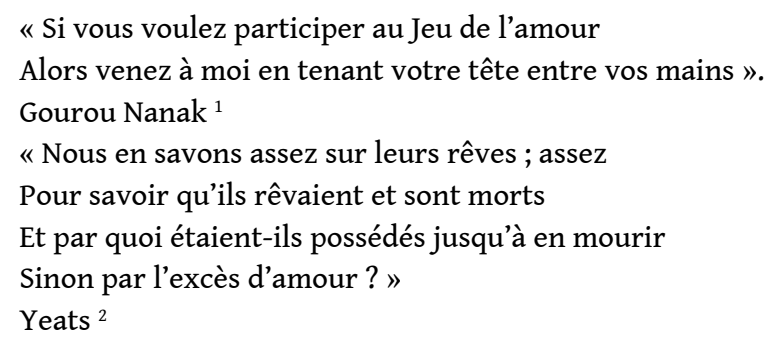

D'un point de vue étymologique, le shahid sikh, comme le martyr chrétien et musulman, est un individu qui « porte témoignage » dans la mort volontaire. L'offrande de la vie se fait ici " protestation symbolique ${ }^{3}$ ", en même temps qu'elle constitue la profession d'une foi à toute épreuve. Le martyr est par essence un acteur politique, qui conteste l'ordre existant et affirme une double transcendance : celle de la loi divine et celle du lien communautaire ${ }^{4}$, que son sacrifice vient ériger en absolus ${ }^{5}$. Dans la plupart des grandes religions, exception faite de l'islam sunnite ${ }^{6}$, le martyr n'est pas le héros qui meurt au champ d'honneur, éventuellement d'une balle perdue ${ }^{7}$. Il en est même l'antithèse, et appartient non pas au registre du drame, mais à celui de la tragédie ${ }^{8}$. L'expérience de la torture est souvent centrale dans la trajectoire sacrificielle des candidats au martyre, puisqu'elle constitue un moment fort de mise à l'épreuve des convictions. La brutalité du tortionnaire vient répondre à la foi inébranlable du candidat au martyre en même temps qu'elle la valide. Elle n'est pas tant destinée à lui tirer des aveux qu'à lui arracher un reniement, qui viendrait dévaloriser ses convictions et permettrait de réaffirmer la transcendance du pouvoir d'Etat aux dépens de celle des valeurs religieuses et du lien communautaire. La mise à mort du candidat au martyre n'est qu'un dernier recours pour le pouvoir ; elle est un 
aveu d'échec puisqu'elle signifie que le croyant ne s'est pas renié et que ses convictions proprement surhumaines ne peuvent être vaincues que par l'attribut ultime du pouvoir souverain, à savoir le droit de retirer la vie. Moment de refondation pour le pouvoir, l'exécution du martyr le contraint à des contorsions rhétoriques qui constituent sa riposte symbolique à la profession de foi provocante du candidat à la sainteté ${ }^{9}$. Loin de réduire ce dernier au silence, l'exécution constitue le point d'orgue de son cheminement discursif et s'accompagne d'une profusion de paroles et d'écrits hagiographiques qui entretiennent sa mémoire et viennent galvaniser ses coreligionnaires. Les trajectoires sacrificielles individuelles s'effacent alors dans les stratégies martyrologiques déployées par les entrepreneurs identitaires. A travers ces discours hagiographiques, le martyr devient un artefact politique. Sa dépouille suppliciée vient témoigner de la brutalité de l'adversaire étatique et conforter le sentiment d'appartenance commune du groupe, sanctifié par ces morts glorieuses.

2 L'histoire récente du mouvement ethnonationaliste sikh illustre de manière exemplaire cette ambivalence du martyre. Au cours de la première phase du mouvement pour le Khalistan (1984-1990), les guérilleros sikhs étaient animés d'un ardent désir de mort, profondément individualiste, désir s'opposant à la mise au point d'une stratégie de guérilla efficace. Alors que les leaders insurgés incitaient leurs militants à la prudence, ces derniers se ruaient à la mort sans se soucier d'inscrire leur combat dans la durée. $\mathrm{Au}$ cours de cette première phase, les pulsions martyropathes des combattants ont donc contrarié les projets de leurs chefs. Au début des années 1990, les leaders des groupes armés sont parvenus à canaliser ces pulsions de mort, dans le cadre d'une stratégie offensive mieux coordonnée, témoignant de l'influence croissante des services de renseignement pakistanais. Les bardes sikhs (les dhadis ${ }^{10}$ ) ont très tôt insisté sur la dimension nationaliste de ces pratiques sacrificielles. Mais ce n'est que dans un troisième temps, celui de la commémoration de la lutte armée (depuis 1995), que les idéologues du mouvement pour le Khalistan ont, à leur tour, cherché à convertir ces sacrifices individualistes en témoignages politiques au pouvoir performatif : celui de redéfinir les frontières et les inimitiés de la communauté sikhe (le Panth). La construction politique de ces pratiques sacrificielles n'a donc pris un caractère systématique, en particulier grâce à l'Internet, qu'à partir du moment où le mouvement armé a commencé à s'essouffler au Pendjab. Cet usage stratégique des martyrs du Khalistan par les « cyber-khalistanis » a alors servi à commémorer la lutte armée, bien plus qu'à la relancer.

L'invention de l'idiome du martyre sikh

3 Pour bien saisir les enjeux des trajectoires sacrificielles et des pratiques de commémoration des shuhada du Khalistan, il nous faut brièvement rappeler les origines de "l'idiome du martyre " sikh, c'est-à-dire des codes discursifs et esthétiques qui, dans cette population, encadrent la pratique du sacrifice suprême et édictent les (bonnes) manières de le pratiquer et d'en parler ${ }^{11}$. Cet idiome du martyre s'appuie sur des événements historiques mythifiés autant que sur des sentences religieuses continuellement réinterprétées, qui ont conjointement servi l'invention d'une «tradition» sikhe du martyre. Comme nous le verrons, les codes entourant la pratique et l'évocation de la mort polémique chez les Sikhs sont d'une grande flexibilité, ce qui a permis aux entrepreneurs identitaires khalistanis de décerner le titre prestigieux de «shahid» à leurs partisans décédés accidentellement ${ }^{12}$. 
Comme le souligne le sociologue J.P.-S. Uberoi, depuis l'époque de Gourou Nanak, fondateur de cette religion hybride, le sikhisme est « pratiquement autant un mode de vie qu'une manière de mourir ${ }^{13}$ ". L'acceptation stoïque de la mort est au cœur de l'enseignement de Nanak, qui aurait un jour déclaré à ses disciples : «Si vous voulez participer au Jeu de l'amour, alors venez à moi en tenant votre tête entre vos mains ${ }^{14}$ ». Plusieurs épisodes célèbres de l'histoire sikhe ont popularisé cette formule, interprétée de manière littérale dans la tradition populaire dont s'est nourri le mouvement khalistani. Ainsi la légende veut-elle qu'après sa décapitation par un soldat afghan, Baba Deep Singh, l'une des grandes figures de la résistance sikhe au pouvoir moghol, ait porté sa tête entre ses mains pour la déposer dans l'enceinte sacrée du Temple d'or d'Amritsar. Peu avant son martyre, au cours de l'opération «Bluestar» de juin 1984 destinée à le déloger du Temple d'or, le principal inspirateur du mouvement pour le Khalistan, Sant Jarnail Singh Bhindranwale, se référait fréquemment à cet épisode légendaire. «Baba Deep Singh a dû porter sa tête sur plusieurs kilomètres pour la déposer ici, alors que j'ai le privilège de pouvoir offrir la mienne ici même », aurait-il ainsi confié à son entourage peu avant sa mort ${ }^{15}$.

5 Le Panth connut son premier martyr au début du xviie siècle, avec la mort sous la torture du cinquième Gourou, Arjun, en $1606{ }^{16}$. Son acceptation sereine de la mort fut interprétée par ses contemporains comme un acte résolument politique, provoquant le pouvoir moghol pour l'inciter à se réformer ou à dévoiler sa brutalité et son iniquité ${ }^{17}$. Le martyre d'Arjun est commémoré chaque année par les membres du Panth, à l'occasion du Shahidi Divas (« le jour du martyr »). Sa mémoire est également honorée quotidiennement dans la prière de l'ardas, ainsi que celle des martyrs postérieurs. Parmi ces derniers, les plus célèbres sont le neuvième Gourou, Tegh Bahadur, décapité en 1675, Fateh et Jujhar Singh, les deux fils du dixième et dernier Gourou incarné, Gobind Singh, dont le vice-roi de Sirhind ordonna qu'ils soient emmurés vivants en 1705. Banda Bahadur fut, quant à lui, soumis à un supplice particulièrement atroce en $1716^{18}$, à l'instar de Bhai Mani Singh en $1738{ }^{19}$.

6 Bien que le terme arabe "shahid » ait été en usage en Inde depuis le xe siècle, il n'a fait sa première apparition dans la littérature consacrée aux martyrs sikhs qu'au début du xixe siècle. L'historien Louis $\mathrm{E}$. Fenech a ainsi repéré sa première occurrence dans le Shahid-bilas ( Le plaisir du martyr ») de Seva Singh Kaushish ${ }^{20}$. Ce texte, publié autour de 1802, est une hagiographie de Bhai Mani Singh, le célèbre compagnon du Gourou Gobind Singh. Bien que le terme utilisé ici par Seva Singh soit emprunté à l'arabe, il prend une signification différente de celle que lui connaissent les musulmans, notamment les sunnites. Chez ces derniers, la shahadat est le résultat d'un décès accidentel, au combat principalement mais aussi en pèlerinage ou en couches, c'est-àdire au cours d'un acte méritoire ${ }^{21}$. Dans l'islam doloriste chiite, au contraire, le martyre résulte d'un acte intentionnel, qui témoigne de la foi du martyr et de l'iniquité de son adversaire. Ces conceptions chiites de la shahadat se rapprochent de l'idiome du martyre sikh forgé à la fin du xixe siècle. Si l'on peut ici repérer une analogie entre le sikhisme et le chiisme, celle-ci ne suffit pas à étayer l'hypothèse d'une influence chiite sur l'idiome du martyr sikh. Certains historiens ont en revanche relevé des emprunts à l'hindouisme par les concepteurs de la "tradition » sikhe du martyre ${ }^{22}$. A la fin du xixe siècle, ces derniers ont en effet popularisé la notion de "martyre vivant " (zinda shahid), qui s'inspirait de la figure du jivan mukta hindou. Mais alors que le jivan mukhta parvient à atteindre la libération du cycle des réincarnations en se détachant 
du monde matériel, le zinda shahid sikh est résolument engagé dans les affaires d'icibas et c'est à sa résistance à l'oppression qu'il doit son exemplarité.

7 Le mouvement de réforme socioreligieuse des Singh Sabhas, qui a redéfini l'identité panthique et posé les bases du nationalisme sikh à la fin du xixe siècle ${ }^{23}$, a joué un rôle décisif dans la formalisation de cet idiome du martyre sikh. L'invention d'une «tradition» du martyre par les Singh Sabhas a aidé ces réformateurs à souligner l'originalité du sikhisme par rapport à l'hindouisme, mais aussi à légitimer leurs innovations en établissant une continuité entre les martyrs historiques du sikhisme et les sikhs « réformés » (les Khalsa Sikhs ${ }^{24}$ ). Le zinda shahid devint ainsi le type idéal du Khalsa Sikh militant, prêt à endurer l'enfermement, la torture et la mort pour défendre ses convictions personnelles, les valeurs de sa communauté et, ultimement, la justice universelle. C'est cette conception du shahid qui a été transmise aux révolutionnaires du Ghadr ${ }^{25}$, aux nationalistes akalis, aux maoïstes « naxalites » puis aux sécessionnistes khalistanis, et qui se trouve entretenue par les chants des dhadis. Bien que le gouvernement indien ait interdit à ces derniers d'honorer la mémoire des victimes de l'opération "Bluestar ", ils ont souvent évoqué les faits d'armes de Bhindranwale et de Shahbeg Singh dans leurs compositions, en les présentant comme des martyrs. Le titre de shahid leur a d'ailleurs été officiellement décerné par le jathedar de l'Akal Takht ${ }^{26}$, Joginder Singh Vedanti, à l'occasion de la commémoration du 19e anniversaire de l'opération « Bluestar », le 6 juin 2003.

8 Alors qu'il s'appliquait initialement aux martyrs religieux qui ont refusé de se convertir à l'islam, et qui ont connu, de ce fait, des morts douloureuses, le terme « shahid » en est venu à inclure certains «bandits sociaux» (kharku), tels Sucha Singh Surma et Jeuna Maur, ainsi que les soldats sikhs tombés au cours des deux conflits mondiaux et des guerres indo-pakistanaises, ou encore les «terroristes ${ }^{27}$ » Bhagat Singh et Udham Singh, qui avaient pourtant renié leur identité sikhe ${ }^{28}$. Plus récemment, les insurgés khalistanis ont récupéré ce terme pour l'appliquer à leurs camarades morts sous la torture mais également au cours d'accrochages - parfois fictifs - avec les services de sécurité indiens. Cet élargissement sémantique du terme brouille sa spécificité par rapport à celui de " héros ", dont il est pourtant originellement antithétique. Il ne s'agit pas là d'un phénomène propre aux sikhs. On peut en effet observer cette routinisation de l'» idiome du martyre » dans toute l'Asie du Sud, du fait de groupes armés comme de pouvoirs étatiques en quête de légitimation symbolique ${ }^{29}$.

9 La production discursive qui a accompagné la construction d'une «tradition » sikhe du martyre s'est doublée d'une production esthétique qualifiée par l'anthropologue Cynthia Keppley-Mahmood d'» art du massacre ${ }^{30}$ ». Cette forme d'art décrit, avec force réalisme, les tortures infligées aux sikhs candidats au martyre. Ces tableaux ${ }^{31}$, qui ornent les murs de tous les gurudwaras en Inde comme à l'étranger, opposent généralement au visage sévère, cruel et parfois grotesque des bourreaux musulmans, la figure sereine ou extatique des martyrs sikhs. Ces représentations picturales induisent que la foi des shuhada leur donne le pouvoir de dominer la souffrance, voire de la commuer en plaisir intense. La légende veut ainsi que, lorsque ses bourreaux lui demandèrent quelle était sa dernière volonté, Bhai Mati Dass, condamné au supplice de la scie en 1732 après avoir refusé de se convertir à l'islam, ait déclaré : « Lorsque je serai attaché et scié en deux, je souhaiterais que mon visage soit tourné vers mon maître [murshid], afin que je puisse le contempler et qu'il puisse me voir jusqu'à mon dernier souffle. Il peut être certain que j'avancerai avec plaisir vers ma destination finale ${ }^{32}$ ». 
Son contemporain Bhai Sati Dass aurait quant à lui déclaré, au moment de rendre son dernier souffle et après avoir été roulé dans une balle de coton à laquelle on mit le feu : "Seuls ceux qui ont aimé ont atteint Dieu ${ }^{33}$ ". L'un des compagnons de Gourou Teg Bahadur, mis à mort peu avant lui par le supplice de l'ébullition, aurait, pour sa part, déclaré avant de mourir : «Mes compagnons se sont moqués de la mort et de la douleur et se sont fondus à l'Etre suprême. Hâtez-vous et que mon âme s'unisse rapidement au Seigneur ${ }^{34}$ ». Inspiré par ces récits populaires, dont la mémoire est entretenue depuis l'époque des Gourous par les dhadis, l'» art du massacre » sikh cherche à montrer cet amour supposé irradier des martyrs dans leurs derniers instants ${ }^{35}$. Le stoïcisme des suppliciés, attesté par l'immobilité et la rectitude de leur corps, n'en fait pas des figures austères : leur regard défiant ou énamouré vient rappeler qu'ils vont joyeusement à la mort, par amour pour leur Dieu et pour leurs frères. La charge émotive de cette iconographie omniprésente dans les lieux de culte sikhs est donc double : elle produit un sentiment d'effroi par la représentation crue de supplices, mais suscite également l'admiration des fidèles et conforte leur sentiment d'appartenance au Panth, sanctifié et promis à la victoire par ces morts joyeuses ${ }^{36}$. A ce titre, cet " art du massacre » sikh, dont les "cyber-khalistanis » ont fait un usage intense sur l'Internet, présente une dimension performative évidente, en contribuant à faire advenir cette communauté religieuse et politique sikhe dont il prédit la victoire ultime en dépeignant ses souffrances sublimes ${ }^{37}$.

Profil sociologique et trajectoires sacrificielles des martyrs pour le Khalistan

En Inde comme dans les Etats de résidence des sikhs expatriés, les "événements critiques ${ }^{38}$ » de 1984 - attaque de l'armée indienne contre le Temple d'or d'Amritsar, déclenchement d'une campagne de répression dans les campagnes du Pendjab, massacres anti-sikhs dans le nord de l'Inde suite à l'assassinat d'Indira Gandhi par ses gardes du corps sikhs - ont profondément bouleversé les membres du Panth, en causant un « choc moral ${ }^{39}$ » qui s'est répercuté sur leurs modes d'identification, puis sur leurs mobilisations politiques. Les événements de 1984 et le « choc moral » qui en a résulté parmi les sikhs du Pendjab et de l'étranger ont favorisé leur régression sur une "identité-masse", caractéristique de ces situations de fortes tensions sociales et psychologiques alimentant des formes de «stress collectif » propices au développement d'assignations identitaires qui « réduisent la complexité des allégeances multiples à de puissants stéréotypes émotionnels ${ }^{40}$ ».

11 La routinisation de cette terreur d'Etat est apparue dès les années 1970, à l'initiative des élites provinciales, justifiant le recours aux mesures d'exception par la menace qu'aurait fait peser le maoïsme révolutionnaire sur le système politique du Pendjab. Au cours de la décennie suivante, l'Etat fédéral a institutionnalisé ces mesures exceptionnelles dans sa répression du mouvement khalistani, à travers l'adoption de "lois scélérates ${ }^{41}$ " («black laws») qui ont donné carte blanche aux services de sécurité dans leur répression du mouvement séparatiste, aussi bien en matière d'interpellation que de techniques d'interrogatoire, et en dernier recours d'élimination physique (cette répression a peut-être fait plusieurs dizaines de milliers de victimes, mais les rares défenseurs des droits de l'Homme qui ont cherché à préciser ce chiffre ont été à leur tour assassinés).

12 La généralisation de la torture qu'a rendue possible l'institutionnalisation de cet état d'exception a joué un rôle décisif dans la radicalisation des jeunes nationalistes sikhs. En portant atteinte à l'intégrité du corps sikh, et notamment aux symboles identitaires 
associés à cette communauté (le turban et la chevelure, systématiquement profanés par les tortionnaires), les services de sécurité opérant dans la province (la police, les unités paramilitaires et l'armée régulière) ont provoqué un sentiment d'outrage dans l'ensemble de la population sikhe tout en renforçant les convictions politiques de leurs victimes. Pour tous les militants khalistanis qui y ont survécu, cette expérience de la torture a joué un rôle fondateur. Dans les récits qu'ils en font, les militants khalistanis décrivent ces séances de torture comme une épreuve intérieure, où la relation avec le bourreau perd de son importance par rapport à la relation à soi et à Dieu. Ce moment critique relève pourtant de l'ordre politique et non mystique, dans la mesure où il vient mettre en jeu les convictions personnelles et la loyauté au groupe. Les vétérans de la lutte armée ont généralement tendance à minimiser a posteriori les souffrances endurées au cours de ces séances de torture, en conformité avec la tradition du «bol bala ", cet art de la litote dans l'évocation des souffrances morales et physiques du Panth, qui fait la spécificité de l'idiome du martyre sikh ${ }^{42}$. «Certains sont morts de ces tortures, mais pour moi la douleur n'était pas si terrible [...] », nous a ainsi confié un militant des droits de l'Homme proche de la guérilla ${ }^{43}$. Certains vétérans évoquent l'» énergie » qui les animait alors, le terme qu'ils utilisent («shakti ») étant emprunté à l'hindouisme, dans lequel il est associé à la puissance destructrice des déesses martiales (Kali, Durga, etc.). Ainsi une ancienne militante de la Bhindranwale Tigers Force of Khalistan (BTFK), nous a-t-elle confié :

«Tout au long de mon engagement [au sein de la BTFK], j'étais animée par une sorte d'énergie [shakti]. En règle générale, je suis pourtant une personne assez frêle, mais lorsque j'ai été arrêtée et torturée, je n'éprouvais aucune crainte de la douleur. Au cours de mon second séjour en prison, j'ai été torturée sauvagement, on m'a fait subir des électrochocs sur tout le corps, mais les policiers qui me torturaient devaient me supplier de crier, pour ne pas se faire réprimander par leurs supérieurs ${ }^{44} »$.

Si cette exposition personnelle à la terreur d'Etat contribua au passage à l'acte violent des insurgés khalistanis, cette explication par la répression doit cependant être nuancée par la prise en compte des facteurs prédisposant à un tel engagement radical. De fait, tous les jeunes sikh(e)s n'ont pas réagi de manière analogue aux événements critiques de 1984. Certes, de nombreux sikhs sans passé militant ont apporté un soutien moral au mouvement khalistani au lendemain de l'opération "Bluestar », en particulier dans la diaspora. Mais au Pendjab, le mouvement insurgé a trouvé ses principaux appuis dans les rangs des jeunes baptisés et pratiquants (amritdharis), de caste jat et préalablement politisés au sein des syndicats étudiants proches de Bhindranwale, en particulier de la All India Sikh Students Federation (AISSF). La petite paysannerie jat du Majha ${ }^{45}$ a constitué en particulier un important vivier de recrutement pour les groupes armés khalistanis, qui, pour la plupart d'entre eux (à l'exception du Babbar Khalsa, organisation néo-fondamentaliste fondée en 1978 autour d'un agenda plus religieux que politique), sont apparus dans la seconde moitié des années 1980. Comme l'a relevé Hamish Telford, la majorité des militants khalistanis des années 1980-1990 étaient originaires de deux districts du Majha, Gurdaspur et Amritsar, qui se classaient respectivement au huitième et au neuvième rang des douze districts du Pendjab en termes de revenus agricoles ${ }^{46}$. C'est déjà dans cette petite paysannerie que Bhindranwale avait trouvé ses principaux soutiens. La paysannerie jat du Majha avait moins profité de la "Révolution verte» des années 1960-1970 que les fermiers capitalistes du Malwa ${ }^{47}$, et elle avait trouvé en Bhindranwale un nouveau porte-parole traduisant ses frustrations socio-économiques dans l'idiome du religieux. A ce terreau 
social favorable s'ajoutait un atout géopolitique. Par leur situation géographique, frontalière du Pakistan, les districts de Gurdaspur et d'Amritsar étaient en effet des terrains favorables à la lutte armée, offrant la possibilité d'un repli tactique vers le sanctuaire pakistanais. Les jeunes sikhs de ces districts connaissaient bien les chemins de contrebandiers permettant de franchir la frontière en déjouant la vigilance des forces de sécurité indiennes. De surcroît, certains garde-frontières sikhs apportèrent leur soutien aux insurgés, lorsqu'ils ne rejoignirent pas leurs rangs.

D'autres facteurs, plus inattendus, ont joué un rôle crucial dans l'engagement au sein du mouvement armé khalistani. Pour les hommes comme pour les femmes qui y participèrent ${ }^{48}$, cet engagement politique violent fut souvent vécu comme un jeu avec la mort, qui, comme toute conduite à risque, impliquait une "mise en jeu de soi ${ }^{49}$ ". Cette dimension ludique ${ }^{50}$ s'est trouvée confirmée, dans le cas sikh, par les entretiens que nous avons pu conduire auprès de vétérans des groupes armés khalistanis. Ceux-ci mettent tous en avant le caractère exaltant de leur expérience militaire, qui transparaît notamment dans les propos des anciennes combattantes, animées par un «féminisme martial » analogue à celui des combattantes tamoules du LTTE ${ }^{51}$. L'ancienne militante de la BTFK précitée, aujourd'hui mère de famille, déplore ainsi la banalité désolante de son existence depuis sa démobilisation. Elle se plaint de ne plus trouver l'énergie d'aller jusqu'au bazar pour y effectuer ses achats quotidiens, alors que, lorsqu'elle partageait la vie des militants, elle affirme qu'elle pouvait marcher sans difficulté des jours durant ${ }^{52}$. L'étude de Harish K. Puri, Paramjit Singh Judge et Jagrup Singh Sekhon met également en lumière, à partir d'un échantillon plus substantiel, la dimension ludique de l'engagement dans les groupes armés khalistanis. Sur les trois cent vingt-trois « terroristes » dont les auteurs ont pu reconstituer la " carrière », $38 \%$ auraient rejoint les groupes armés pour le "fun ", $12 \%$ pour mener en toute impunité des activités criminelles, $10 \%$ du fait de leurs contacts directs avec des «terroristes », et seulement $5 \%$ pour contribuer à l'avènement du Khalistan, ces militants « idéologiques » étant à peine plus nombreux que ceux ayant rejoint la guérilla pour accomplir ou pour échapper à une vendetta familiale ${ }^{53}$.

On retrouve en fait dans le mouvement armé pour le Khalistan les trois types-idéaux de l'insurgé religieux, identifiés par Farhad Khosrokhavar dans son étude ${ }^{54}$ des Bassidji iraniens (le « ludique », l'» opportuniste » et le « martyropathe »), et dont Amélie Blom a récemment montré l'intérêt dans l'analyse des trajectoires individuelles conduisant à l'engagement au sein des organisations jihadistes actives au Cachemire indien ${ }^{55}$. L'engagement de milliers de jeunes sikh(e)s au sein des groupes armés qui apparurent à partir de l'été 1984 s'explique lui aussi par l'effet de séduction du jeu violent auquel il permettait de s'adonner, par les stratégies d'accumulation qu'il rendait possibles, et par la volonté de ces jeunes garçons et filles de retrouver dans la mort le contrôle de leur existence, qui leur était quotidiennement dénié par la terreur d'Etat ${ }^{56}$.

Logiques sacrificielles contre stratégie insurrectionnelle : les premières années de la lutte armée

16 A l'instar des chebab palestiniens de la première Intifada, les premiers combattants khalistanis étaient prêts à assumer le risque de mort en combattant leur adversaire, et si certains d'entre eux étaient animés d'un puissant désir de mort préalablement à leur engagement au sein des groupes armés, aucun ne songea pour autant à se livrer à des attentats-suicides sur des cibles civiles ou militaires. Les jeunes sikh(e)s aspirant à la shahadat espéraient tomber les armes à la main, à l'instar des combattants du jihad, et 
ceux ou celles qui n'y sont pas parvenus en dépit de tous leurs efforts, se sont résignés à vivre dans l'amertume. A ce titre, les trajectoires sacrificielles des premiers combattants khalistanis se situent à mi-chemin entre le modèle optimiste du martyre porté par la première Intifada et celui, résolument pessimiste, des groupes «martyropathes » iraniens, tels les Bassidji, dont les membres ne se souciaient plus de vivre mais aspiraient à la destruction de leur ennemi par l'anéantissement de leur personne. Le cas sikh présente des affinités avec chacun de ces types-idéaux du martyre moderne, en fusionnant sentiment national et affects religieux. L'attaque de l'armée indienne contre le Temple d'or a en effet convaincu la jeunesse sikhe que la menace posée par le "gouvernement de brahmanes » de New Delhi sur l'avenir du Panth était de nature politique et religieuse, les autorités centrales se montrant hostiles à l'autonomie et à la prospérité de la nation panthique, tout autant qu'à l'émancipation des sikhs vis-à-vis de l'hindouisme. Comme leurs homologues palestiniens, les candidats sikhs au martyre ont donc combattu pour libérer leur nation et leur religion 57 .

17 Si le martyre khalistani se distingue du mouvement martyropathe animé par le Bassidje iranien ou du « néo-martyre » d'Al-Qaïda ${ }^{58}$, ces différences tiennent moins à des motifs idéologiques divergents qu'à des choix stratégiques distincts. La spécificité du martyre khalistani, par rapport à ses équivalents sunnites et chiites contemporains, découle moins de sa dimension nationaliste que de son absence de portée stratégique ${ }^{59}$. Ce caractère a-stratégique du martyre khalistani résulte de la nature non instrumentale de l'acte résistant dans le mouvement pour le Khalistan, c'est-à-dire de « son autonomie à l'égard des objectifs stratégiques, politiques ou militaires, définis rationnellement ${ }^{60}$ ». A la différence des leaders du Bassidje et du Hamas, ou plus récemment, des Tigres tamouls, d'Al-Qaïda et des groupes combattants tchétchènes, les commandants des groupes armés sikhs n'ont jamais développé d'» idéologie sacrificielle ${ }^{61}$ » pour convertir les pulsions mortifères de leurs militants en une arme de guerre. L'irrépressible désir de mort de nombreux combattants khalistanis est même venu entraver l'élaboration d'une stratégie coordonnée par les chefs des groupes armés, en produisant plus de martyrs que de survivants, comme en témoigne ce militant chargé de l'entraînement des combattants dans un camp pakistanais à la fin des années 1980 :

«Le problème [des sikhs] c'est que, comme les Iraniens, nous avons cette p... de tradition du martyre... Il fallait être là-bas [au Pakistan] pour le comprendre. Nous avons perdu la tête... Parce qu'il faut vivre, mec, il faut vivre... Parfois, j'étais moimême comme hypnotisé et ma femme avait peur que je ne revienne pas [à Londres]. Parce que c'était si facile de passer de l'autre côté... [...] Le problème, avec le martyre, c'est que tout le monde veut y accéder et que tout ça finit par devenir une affaire de prestige individuel ${ }^{62} »$.

Dans les premières années du mouvement pour le Khalistan (1984-1986), qui n'était initialement qu'une riposte symbolique à la répression indienne, les jeunes combattants sikhs adoptèrent une pratique martiale profondément individualiste: celle du wanggar. Cette pratique consistait à « informer et défier » l'adversaire, pour le provoquer en duel. Les jeunes combattants se rendaient ainsi individuellement devant les thanas (commissariats) pour y abreuver d'injures leur cible, et pour lui communiquer l'heure à laquelle elle serait attaquée. Ce comportement "chevaleresque» comportait une dimension ludique mais également politique, puisqu'il permettait aux rebelles sikhs de réfuter les accusations de leur adversaire étatique indien, qui les présentait comme des «terroristes» à la solde du Pakistan. 
L'exemple le plus fameux de wanggar est celui d'Avtar Singh, un commandant insurgé du village de Brahma, qui, dans la nuit du 27 au 28 juin 1986, s'adressa par mégaphone aux paramilitaires de la Central Reserve Police Force (CRPF) cernant son village, pour les défier de l'interpeller sans porter préjudice aux villageois ${ }^{63}$.

19 Au-delà du caractère ludique de ces provocations, les premiers insurgés khalistanis étaient guidés par des pulsions mortifères mêlant désir d'annihilation de soi et affirmation individualiste. Cet ardent désir de mort des guérilleros khalistanis, dont les leaders insurgés eux-mêmes soulignent le caractère profondément individualiste, s'est trouvé confirmé par nos entretiens avec des rescapés de la lutte armée des années 1984-1995. Privés d'avenir par l'institutionnalisation de la terreur d'Etat dans la province, ces jeunes martyropathes ont cherché à retrouver le contrôle de leur existence, et par là même la liberté et la dignité, en choisissant de mourir, comme en témoigne une ancienne militante du Babbar Khalsa :

«J'ai rejoint les militants parce qu'après l'attaque contre le Temple d'or, nous n'étions plus autorisés à nous rendre à Amritsar et j'ai commencé à me sentir tellement désespérée que je décidai de me suicider. Je voulus prendre du poison et j'avais tout préparé, mais au moment où j'attrapai la bouteille, je réalisai qu'elle était enveloppée dans du papier journal, et je remarquai un article sur le suicide qui expliquait à quel point c'est un acte égoïste et sacrilège. C'est alors que je décidai que, plutôt que de mourir comme ça, je pourrais mourir par amour pour ma communauté. Après, je suis allée trouver les babbars et j'ai épousé l'un d'entre eux pour pouvoir participer à la lutte armée ${ }^{64} »$.

Ces propos suggèrent l'ampleur du traumatisme occasionné par les événements de 1984 dans la jeunesse sikhe, au sein de laquelle ils ont provoqué un « choc moral » mortifère. Le désir de mort, profondément individualiste, dont témoigne ici cette combattante de la première heure, s'est cependant trouvé réorienté vers un projet altruiste. L'amour de la mort des premiers combattants sikhs s'est ainsi commué en un amour jusqu'à la mort. Cette formulation semblera peut-être trop imagée, mais elle traduit bien la relation affective, voire érotique, que les combattants sikhs entretenaient avec la mort. Sant Jarnail Singh Bhindranwale, mort en martyr au cours de l'opération "Bluestar ", avait l'habitude de recourir à des licences poétiques pour évoquer sa mort annoncée. Parmi ces métaphores, la plus courante était celle du baiser à la corde du pendu (fansi). Cette image a par la suite été rendue célèbre par les chansons populaires exaltant le combat sécessionniste. Dans l'une de ces chansons, Wah Nee Azadhia ${ }^{65}$, composée par un musicien sikh expatrié en Allemagne, on trouve par exemple ce couplet: "Nous avons embrassé la corde du pendu / et d'innombrables vies s'y sont balancées ». De manière plus éloquente encore, un autre couplet de cette chanson décrit l'engagement nationaliste comme un mariage avec la mort : « Notre amour pour toi [l'indépendance] nous rend fous de passion [shudai]. Nous ne pouvons supporter d'être séparés de toi. Un mot de toi et nous sommes prêts à prononcer les voux du mariage [lama] avec la Mort ${ }^{66} »$. Les termes pendjabis utilisés par l'auteur sont sans équivoque : il s'agit bien ici d'une passion amoureuse, évoquant explicitement la réconciliation d'Eros et Thanatos. Paraphrasant Yeats, l'anthropologue Cynthia Keppley-Mahmood en arrive alors à la conclusion que les martyrs sikhs " moururent d'un excès d'amour ${ }^{67}$ ». Les pertes considérables en vies humaines ${ }^{68}$ occasionnées par ces pratiques sacrificielles ont incité les leaders des groupes armés khalistanis à adopter une stratégie offensive mieux coordonnée, impliquant une prise de risque plus limitée pour les combattants. Afin d'assurer la survie du mouvement, il était devenu essentiel de 
convaincre les militants de l'importance des résultats de leur acte sacrificiel, qui avait jusqu'alors sa propre finalité parmi les résistants khalistanis ${ }^{69}$. Jusqu'au début des années 1990, le mouvement insurrectionnel khalistani s'est démarqué par son caractère pré-organisationnel. Malgré plusieurs tentatives pour doter les groupes armés d'un Etat-major (le comité panthique), ces projets ont été tenus en échec par l'individualisme des militants contestant l'autorité de leurs commandants, autant que par le factionnalisme du mouvement. Le premier comité panthique, formé en 1986, a rapidement implosé et a été concurrencé par un second comité, apparu en 1988 autour des groupes néo-fondamentalistes soutenus par le Pakistan. Au sein de chaque organisation, les défections étaient fréquentes, les militants quittant régulièrement leur groupe pour s'affilier à une autre organisation ou pour fonder la leur. Ce n'est qu'au début des années 1990, sous l'influence des services de renseignement pakistanais, que les groupes armés khalistanis, en particulier la Khalistan Commando Force (KCF), ont réussi à se doter de structures organisationnelles de type cellulaire. Cette structure cellulaire était destinée à mieux coordonner les activités des militants, tout en leur permettant de se prémunir contre l'infiltration des services de sécurité indiens. Les services de renseignement pakistanais, en particulier l'Inter Services Intelligence (ISI), ont également incité les insurgés sikhs à maximiser les résultats de leurs actions tout en minimisant leur prise de risque, en se concentrant sur des cibles économiques et en faisant usage d'explosifs plutôt que d'armes à feu, plus difficiles à transporter et à camoufler ${ }^{70}$. Ce n'est pourtant qu'à l'issue de la défaite militaire de la guérilla au Pendjab, aux alentours de 1993, que les agents de l'ISI ont véritablement pris le contrôle des opérations des groupes sikhs, en les incitant désormais à se livrer à des actions terroristes sur des cibles civiles ou à des assassinats ciblés de personnalités ${ }^{71}$. L'impact de cette "reprise en main » des groupes armés sikhs par l'ISI ne doit pourtant pas être surévalué. Contrairement à ce qui a pu se passer sur d'autres terrains (notamment au Cachemire), c'est dans les premières années du mouvement qu'on a recensé le plus grand nombre de pratiques sacrificielles chez les combattants sikhs, sans que les leaders des groupes armés et les services de renseignement pakistanais n'aient eu la moindre responsabilité dans ces morts volontaires. De surcroît, lorsque l'ISI a cherché à pousser les khalistanis à perpétrer des attentats-suicides, au début des années 1990, ses officiers se sont heurtés à un refus catégorique, leurs "protégés » se justifiant par l'incompatibilité du suicide avec les dogmes sikhs ${ }^{72}$. Les combattants sikhs ne se sont donc jamais alignés sur la vulgate kamikaze et leurs trajectoires sacrificielles détonnent singulièrement dans le paysage insurrectionnel contemporain. La commémoration des shuhada du mouvement pour le Khalistan

La dimension anomique et individualiste des trajectoires sacrificielles des combattants khalistanis n'apparaît naturellement dans aucune des "nécrologies hagiographiques ${ }^{73}$ » consacrées aux shuhada khalistanis. La dimension profondément individualiste de ces pratiques du martyre entre en effet en contradiction avec le projet de construction nationale auquel se sont attelés les idéologues khalistanis. La commémoration des martyrs khalistanis a donc vertu à faire de ces suicides anomiques à peine voilés des sacrifices altruistes dépourvus de toute ambiguïté. A travers cette reconstruction politique des sacrifices individuels des combattants khalistanis, les idéologues du mouvement ont également cherché à transformer une série d'échecs tactiques en un atout stratégique, en faisant de ces morts individuelles « une ressource, une forme de pouvoir symbolique ou de capital politique ${ }^{74} \gg$. 

de cas, heurtée au problème de son intentionnalité mais sans jamais l'expliciter, comme le montrent les hagiographies consacrées aux martyrs du Khalistan sur l'Internet. Ainsi Bhai Fauja Singh, le plus célèbre des treize sikhs tombés sous les balles des partisans du gourou des Sant Nirankaris le 13 avril 1978, se trouve-t-il qualifié de shahid par les sites Internet saintsoldiers.com et sikhvision.co.uk, alors que son sacrifice ne fut pas intentionnel mais accidentel, puisqu'il ne pouvait prévoir la réaction des Nirankaris et ne se doutait donc pas qu'il allait à la mort. Le texte célébrant la mémoire de Bhai Fauja Singh sur le site saintsoldiers.com affirme pourtant que les treize sikhs abattus par les Nirankaris au cours de cet incident «n'ont pas seulement réveillé le Panth ; ils nous ont rendus plus forts et nous ont incités à être plus vigilants à l'avenir ». Tout en s'inspirant de l'idiome du martyre standardisé par les Singh Sabhas, les « cyber-khalistanis » l'ont donc réinvesti pour donner une nouvelle signification à la shahadat, en en faisant non plus seulement une protestation symbolique au service de la justice, mais également un sacrifice volontaire offert à la nation. Cette reconstruction nationaliste du martyre sikh par les khalistanis répond à un objectif de mobilisation qui se trouve explicité par ces lignes, venant conclure la section consacrée aux martyrs du Khalistan du site saintsoldiers.com : "Après avoir médité sur le passé, nous vous demandons de vous tourner vers le futur: pouvons-nous nous permettre de vivre sous la coupe du gouvernement de Delhi dix années de plus?»

24 A l'instar du Central Sikh Museum d'Amritsar, où les œuvres de Kirpal Singh sont exposées, les sites khalistanis proposant des reproductions des œuvres marquantes de l'» art du massacre » sikh et des photographies des shuhada tombés sous les balles ou sous la torture de l'armée indienne au cours des années 1980 et 1990, «fourni[ssent] un espace où s'arrêter pour contempler l'ardas [la prière récitée deux fois par jour par les sikhs pratiquants et honorant la mémoire des shuhada] ». En d'autres termes, « il s'agit d'un lieu [...] qui permet aux sikhs de recevoir le darshan [la vision] de leurs Gourous, de leurs martyrs héroïques et des artefacts multiples et variés auxquels ils furent associés ${ }^{75}$ \%. La commémoration électronique des shuhada sikhs procède d'un même projet de construction d'une mémoire partagée par l'image que célèbre le musée d'Amritsar. L'anamnèse numérique des khalistanis présente cependant une spécificité : elle s'inscrit dans le cadre d'un projet nationaliste, où le sang des martyrs devient un facteur de cohésion nationale autant que le signe du destin historique de la Sikh Qaum. De fait, pour les sécessionnistes sikhs comme pour Bhindranwale, qui les a inspirés sans toutefois jamais prendre ouvertement position en faveur du Khalistan, le Panth est " une race dont l'histoire est écrite dans le sang des martyrs ${ }^{76}$ ".

25 De même que les dhadhis passent sous silence les tortures physiques subies par les shuhada du Khalistan, afin de ne pas démoraliser leur auditoire ${ }^{77}$, les "cyberkhalistanis " s'abstiennent généralement de représenter les cadavres mutilés des martyrs du Khalistan, ce qui n'exclut pas qu'ils fassent, par ailleurs, usage d'images horrifiques dans leur propagande, comme nous le verrons plus loin. Les concepteurs des sites saintsoldiers.com et sikhworld.co.uk, qui, jusqu'à leur récente fermeture, célèbraient respectivement la mémoire de seize et de vingt-sept martyrs du Khalistan, ont ainsi choisi de représenter leurs sujets encore vivants ${ }^{78}$. Sur les clichés choisis, le regard de ces combattants apparaissait tantôt défiant, évoquant alors la figure du kharku (l'insoumis), tantôt parfaitement détaché, évoquant alors le zinda shahid (ce vivant-mort libéré de tout attachement au monde profane). Les clichés de

Cultures \& Conflits, 63 | automne 2006 
Bhindranwale, de Gurbachan Singh Manochahal ${ }^{79}$ et de Talwinder Singh Parmar ${ }^{80}$ entraient clairement dans la première catégorie, à l'instar de ceux des combattants arborant fièrement leurs armes à feu sur le site Internet sikhworld.co.uk. L'un d'entre eux, Shaheed Bhai Kulwinder Singh, était représenté accroupi, bardé de deux kalachnikovs et pointant un revolver vers le photographe ${ }^{81}$. Le cliché de Gurjrant Singh Budsinghwala ${ }^{82}$ reproduit sur les deux sites Internet mettait, quant à lui, en scène le zinda shahid archétypal, au regard vide, tourné vers son monde intérieur. Le texte qui accompagnait cette photographie sur le site Internet saintsoldiers.com précisait d'ailleurs :

«Bien que Bhai Budsinghwala ait eu entre les mains des millions de roupies, en tant que leader de la Khalistan Liberation Force, l'état de sa maison suffisait à démontrer qu'il n'était pas fait pour devenir un politicien akali [l'auteur sous-entendant ici que l'ensemble des akalis sont corrompus]. Il était au-delà des intérêts matériels. Son engagement au service du Khalistan était sincère et total ».

Si ces deux sites se sont abstenus de représenter les corps mutilés de ces martyrs, tel n'est pas le cas d'un autre site, plus amateur, dont le concepteur a mis en ligne les illustrations accompagnant un calendrier du BKI honorant la mémoire des shuhada de l'organisation. Avant sa fermeture récente, le site sikhvision.com consacrait une page à la mémoire des "martyrs du Khalistan » tombés depuis 1978, c'est-à-dire depuis l'incident entre les Sant Nirankaris et les partisans de Bhindranwale. Dans cette section, huit pages des calendriers de la BKI se trouvaient reproduites. Elles représentaient toutes, dans leur partie gauche, le cliché d'un martyr de la BKI encore vivant et, dans leur partie droite, un cliché de leur cadavre, souvent atrocement mutilé. Entre ces deux images était inséré un médaillon de l'Akal Takht, dans l'état où l'a laissé l'armée indienne lorsqu'elle s'est retirée du complexe du Temple d'or au terme de l'opération «Bluestar ", c'est-à-dire en ruines. Par cette juxtaposition du corps torturé du martyr et du siège effondré du pouvoir temporel panthique, les babbars insistent sur la dimension résolument politique de leur sacrifice. Pour les sécessionnistes sikhs, l'Akal Takht représente moins une institution religieuse que le symbole de la souveraineté panthique, et le sacrifice des shuhada khalistanis s'explique avant tout par leur dévotion à la cause nationale sikhe, elle-même revigorée par ces morts glorieuses.

Bouleversant les codes discursifs encadrant la construction sociale du martyre sikh depuis la fin du xixe siècle pour en faire un instrument de cohésion et de mobilisation nationale, les " cyber-khalistanis » ont également réinventé " l'art du massacre » qui constitue aujourd'hui encore, avec les chants des dhadis, le principal vecteur de transmission du souvenir des martyrs au sein du Panth. La plupart des sites Internet khalistanis accordent une place centrale aux images horrifiques de cadavres anonymes, où se mêlent combattants et civils innocents. Le site Internet saintsoldiers.com comportait ainsi, jusqu'à sa fermeture, une section intitulée "genocide of Sikhs », qui rassemblait dix-huit clichés de corps ensanglantés, pour la plupart ceux de combattants khalistanis, comme en témoignaient les armes qu'ils portaient parfois encore en main ${ }^{83}$. Le site burningpunjab.com, lancé par un sikh de Chandigarh en septembre 1997, s'est pour sa part spécialisé dans la publication en ligne de tels clichés, jusqu'à sa récente fermeture, suite au décès de son créateur, Sukhbir Singh Osan. Si ces clichés s'apparentent à « l'art du massacre » dont nous avons parlé plus haut, ils ont cependant la spécificité de ne représenter que des morts anonymes, sans histoire, dont les visages tuméfiés sont désespérément muets. A la différence des corps mutilés des martyrs 
classiques, ces cadavres sont interchangeables. Ils ne racontent aucune trajectoire individuelle mais témoignent collectivement de la brutalité de l'Etat indien et interpellent de ce fait leurs spectateurs. Ces clichés horrifiques ne sont d'ailleurs pas seulement destinés aux sikhs d'Inde et de l'étranger mais s'adressent également à la " communauté internationale ", par le biais de médias de masse devenus les "témoins globaux du martyre ${ }^{84}$ ».

Souvent publiés dans la section "genocide of the Sikhs" des sites khalistanis, ces clichés interprètent le conflit du Pendjab à partir de la grammaire internationale de la souffrance collective ${ }^{85}$. L'usage de photographies particulièrement crues des martyrs communautaires, mises en scène de manière quasi pornographique ${ }^{86}$, relaie en effet l'emploi du terme "génocide " sur le terrain visuel. Par leur extrême violence, ces clichés ne sont plus destinés à susciter l'admiration des martyrs et le désir d'émulation de ces morts glorieux, à l'instar de « l'art du massacre » formalisé par Kirpal Singh, à la portée à la fois revigorante et incitative, qui vise à entretenir l'optimisme résolu du Panth (résumé par la notion de chardi khala ${ }^{87}$ ). Il s'agit en revanche ici de provoquer l'indignation et de mettre en cause l'humanité du bourreau, autant que celle des spectateurs qui choisiraient la passivité après avoir contemplé ces images. Par cette mise en demeure, ceux qui les observent se voient donc sommés de témoigner des souffrances du peuple sikh, en lieu et place de ces morts anonymes. L'effacement du martyr devant les victimes se traduit alors par un transfert de responsabilité vers le spectateur, auquel se trouve déléguée la charge du témoignage. Par là même, cette iconographie polémique constitue le principe fondateur d'une communauté morale unissant la population victimisée à son public distant.

Le véritable sujet de ces clichés n'est donc plus l'individu martyr et son histoire personnelle, mais bien la violence de l'Etat indien à l'égard de la " nation » sikhe, dont chacun de ces corps porte les stigmates. Le choc émotionnel suscité par ces clichés se substitue à l'administration de la preuve et simplifie à l'extrême les enjeux de la lutte de libération nationale khalistanie en en faisant une question de vie ou de mort. Ce recours aux "politiques de la survie » n'est pas nouveau pour les nationalistes sikhs, puisque les dirigeants akalis l'ont banalisé depuis les années 1940. Il prend cependant ici un caractère systématique et une forme particulièrement brutale, qui somme la " communauté internationale" d'apporter son aide aux sikhs, en même temps qu'il commande à ces derniers de renoncer à leur identité individuelle pour se mobiliser contre l'Etat indien, et ainsi donner un sens aux sacrifices des martyrs. Pour leurs spectateurs sikhs, ces clichés horrifiants ont valeur de «cris de guerre appelant au renoncement à l'existence individuelle», en suggérant que la mort personnelle renforce et revitalise la collectivité et, réciproquement, que l'immortalité des disparus dépend de la survie «de la nation, de la race ou du parti qui, en absorbant avec gratitude leur sacrifice, constituera la réalisation la plus durable des victimes ». Pour Zygmunt Bauman, les stratégies de l'immortalité collective propres aux entreprises identitaires et totalitaires ont ceci de spécifique qu'elles "demandent activement le sacrifice de la vie et glorifient la mort "pour la cause" ${ }^{88}$ ». La commémoration électronique des martyrs du Khalistan ou des victimes anonymes de la terreur d'Etat indienne de la part des "cyber-khalistanis" remplit cette double fonction de désindividualisation, en appelant au renoncement à soi pour la nation et en collectivisant les morts individuelles. L'Internet fournit ainsi un espace de réalisation privilégié pour les «stratégies de la vie " propres aux mouvements nationalistes, où le choc horrifiant des images se substitue au poids argumentatif des mots pour simplifier 
au maximum le message, en le ramenant à une question de survie, tout en élargissant potentiellement son "audience", rendue moralement interdépendante des victimes par le simple fait d'être, à son tour, témoin de leurs souffrances.

Le rôle central tenu par la commémoration des martyrs dans les stratégies de construction et de mobilisation identitaire déployées par les khalistanis dans le cyberespace peut, de prime abord, sembler paradoxal : alors que l'ardent désir de mort des jeunes combattants khalistanis a facilité la victoire militaire de l'armée indienne sur les groupes auxquels ils étaient affiliés, la commémoration électronique de leur sacrifice permet aujourd'hui au mouvement khalistani d'assurer sa survie sur l'Internet. Ce paradoxe disparaît cependant si l'on admet que le martyre vécu et le martyre mis en scène correspondent en fait à deux réalités politiques bien distinctes et parfois contradictoires. Chez les sikhs comme chez les musulmans les plus radicaux ${ }^{89}$, l'expérience du sacrifice suprême est généralement l'aboutissement d'une trajectoire profondément individualiste, mêlant résistance à l'oppression et ardent désir de mort. Le supplicié mis en scène dans le discours et l'esthétique martyrologique est, pour sa part, un artefact politique, une figure exemplaire de détermination stoïque et d'abnégation patriotique, qui ne se rue pas vers la mort mais qui s'y est seulement résigné, au nom des intérêts supérieurs de sa nation et/ou de sa religion.

\section{NOTES}

1.. Nous remercions Christine Moliner, doctorante au Centre d'études de l'Inde et de l'Asie du Sud (CEIAS), pour ses commentaires sur une version préliminaire de ce texte. Slok Varan te Vadhik 20, Adi Granth, cité par Jagdev S.S., Tales of Truth (Sikh Martyrs), Birmingham, Sikh Missionary Resource Centre, 1997 (1re éd. 1991), p. 44.

2.. Yeats W.B., « Easter, 1916 ».

3.. Smith L.B., Fools, Martyrs, Traitors. The Story of Martyrdom in the Western World, Evanston, Northwestern University Press, 1997, p. 12.

4.. Peterson A.L., Martyrdom and the Politics of Religion. Progressive Catholicism in $\mathrm{El}$ Salvador's Civil War, Albany, New York University Press, 1997, p. 15.

5.. Albert J.-P., « Sens et enjeux du martyre : de la religion à la politique », Centlivres P. (dir.), Saints, sainteté et martyre. La fabrication de l'exemplarité, Neuchâtel/Paris, Editions de l'Institut d'ethnologie / Editions de la Maison des sciences de l'homme, 2001, p. 19.

6.. Dans l'islam sunnite, le martyr est avant tout « celui qui atteste de sa foi par la mort reçue en luttant sur la voie de Dieu, le djihad »; Centlivres P., ibid., p. 178.

7.. Sur la distinction entre la figure du héros et celle du martyr, voir Uberoi J.P.-S., Religion, Civil Society and The State. A Study of Sikhism, Delhi, Oxford University Press, 1999, (1re éd. 1996).

8.. Zins M.J., « Morts pour la patrie ! La symbolique de la guerre de Kargil de l'été 1999 et la politique des funérailles du gouvernement nationaliste hindou », Hassner P., Marchal R., (dir.), Guerres et sociétés. Etat et violence après la Guerre froide, Paris, Karthala, 2003, p. 318. 
9.. Sur la « défiguration » des martyrs par le pouvoir qui les met à mort, voir Smith L.B., Fools, Martyrs, Traitors, op. cit., p. 17.

10.. Sur ces artistes engagés, voir Pettigrew J., « Songs of the Sikh Resistance Movement », Asian Music, 23 (1), automne / hiver 1991-1992, pp. 85-118.

11.. Sur le martyre comme " discours ", c'est-à-dire comme « manière de mourir pour Dieu et d'en parler », voir Boyarin D., Dying for God. Martyrdom and the Making of Christianity and Judaism, Stanford, Stanford University Press, 1999, p. 94.

12.. Dans le cas palestinien, Laetitia Bucaille relève également que "le "martyr" est à la fois cette victime innocente et le héros qui risque ou offre sa vie au combat ", voir Bucaille L., "L'impossible stratégie palestinienne du martyre : victimisation et attentat suicide ", Critique internationale, $\mathrm{n}^{\circ} 20$, juillet 2003, p. 119.

13.. Uberoi J.P.-S., Religion, Civil Society and the State, op. cit., p. 90.

14.. "Jao to prem khelan ka chao / Sir dhar tali gali meri ao".

15.. Keppley-Mahmood C., « Playing the Game of Love: Passion and Martyrdom Among Khalistani Sikhs », Pettigrew J., (dir.), Martyrdom and Political Resistance. Essays from Asia and Europe, Amsterdam, VU University Press, 1997, p. 23.

16.. Selon la tradition sikhe, Arjun aurait d'abord été contraint de s'asseoir sur une plaque d'acier chauffée à blanc, pendant qu'on versait du sable brûlant sur son corps ; devant son stoïcisme, le gouverneur de Delhi, Chandu, aurait décidé de lui infliger une autre série de tortures en le plongeant dans l'eau glacée de la rivière Ravi ; voir Jagdev S.S., Tales of Truth, op. cit., pp. 26-27. La véracité de ces récits populaires demeure cependant sujette à caution, certains auteurs allant jusqu'à mettre en doute le martyre d'Arjun ; sur ce point, voir Fenech L.E., Playing the Game of Love. Martyrdom in the Sikh Tradition, Delhi, Oxford University Press, 2000, p. 117 et suivantes.

17.. Uberoi J.P.-S., Religion, Civil Society and the State, op. cit., p. 89.

18.. Après qu'il eût refusé de se convertir à l'islam, on tua son fils devant lui, on aspergea son visage de son sang et on le força à avaler son foie; devant l'obstination de Banda Singh, ses bourreaux lui arrachèrent les yeux puis le découpèrent lentement, membre par membre.

19.. Celui-ci fut également condamné à être démembré après avoir refusé d'abjurer sa foi.

20.. Fenech L.E., Playing the Game of Love, op. cit., p. 7.

21.. Esposito J., (dir.), The Oxford Dictionary of Islam, New York, Oxford University Press, 2003, p. 193.

22.. Fenech L.E., Playing the Game of Love, op. cit., p. 69.

23.. Sur la contribution des Singh Sabhas à la redéfinition de l'identité sikhe, voir Oberoi H., The Construction of Religious Boundaries. Culture, Identity and Diversity in the Sikh Tradition, Delhi, Oxford University Press, 1997.

24.. Fenech L.E., Playing the Game of Love, op. cit., p. 21 et p. 52.

25.. L'un d'entre eux, Mewa Singh, fut déclaré shahid ; il fut exécuté par les autorités canadiennes le 11 janvier 1915 pour avoir abattu un inspecteur de l'immigration en réponse à l'humiliation infligée aux sikhs par ce service au moment de l'affaire du Komagata Maru.

26.. Le jathedar de l'Akal Takht est la plus haute autorité religieuse sikhe, à la tête du "Siège de l'Eternel », cette institution située face au Temple d'or d'Amritsar symbolisant le pouvoir temporel de la communauté sikhe. 
27.. La catégorisation de ces deux personnages est, bien sûr, éminemment polémique ; « terroristes " pour les Britanniques, ils sont aujourd'hui considérés comme des « héros » et des « combattants de la liberté » par la plupart des Indiens.

28.. Fenech L.E., Playing the Game of Love, op. cit., pp. 42-43 ; sur la commémoration du « martyre » d'Udham Singh par la communauté sikhe, voir Fenech L.E., « Contested nationalisms, negotiated terrains: the way Sikhs remember Udham Singh "Shahid" ", Modern Asian Studies, 36 (4), 2002, pp. 827-870.

29.. Sur la genèse d'un « idiome du martyre » nationaliste hindou au cours de la guerre de Kargil, voir Zins M-J., « Morts pour la patrie! », op. cit. ; sur l'usage du terme « shahid » par les insurgés maoïstes népalais, voir Lecomte-Tilouine M., « Kill one, he Becomes one hundred: martyrdom as generative sacrifice in the Nepal's people's war », Social Analysis, à paraître courant 2006.

30.. Keppley-Mahmood C., Playing the Game of Love, op. cit., p. 22.

31.. Le plus célèbre « artiste du massacre » sikh est sans aucun doute Kirpal Singh, dont les oeuvres ont fait l'objet d'innombrables reproductions. Les originaux sont conservés au Central Sikh Museum, situé dans la Clock Tower qui surplombe le Temple d'or d'Amritsar et fondé par Kirpal Singh lui-même.

32.. Cité par Jagdev S.S., Tales of Truth, op. cit., p. 45.

33. «Jin prem kio tin he Prabh payo », ibid., p. 46.

34.. Ibid., p. 49.

35.. Le rôle central du sentiment amoureux dans l'expérience du martyre n'est pas propre aux sikhs. Certains martyrs juifs et chrétiens allèrent gaiement à la mort, non par stoïcisme mais parce qu'ils étaient " passionnément amoureux de Dieu », voir Boyarin D., Dying for God, op. cit., p. 96.

36.. Sur l'impact psychologique produit par les tableaux de Kirpal Singh sur les visiteurs du Sikh Central Museum, voir Fenech L.E., Playing the Game of Love, op. cit., p. 46.

37.. Sur cette dimension « sublime » propre à la topique « esthétique » de la souffrance, voir Boltanski L., La Souffrance à distance. Morale humanitaire, médias et politique, Paris, Métailié, 1993, p. 168.

38.. La notion d'» événement critique » désigne une « rencontre » d'un groupe avec l'autorité, qui conduit à une altération soudaine de sa capacité d'action collective ; voir Gamson W.A., Fireman B., Rytina S., Encounters with Unjust Authority, Homewood, The Dorsey Press, 1982, p. 5.

39.. Jasper J.M., Poulsen J.D., « Recruiting strangers and friends: moral shocks and social networks in animal rights and anti-nuclear protests ", Social Problems, 42(4), 1995, pp. 498-499. Les auteurs définissent ces « chocs moraux » comme des événements ou des informations inattendus, produisant un sentiment d'» outrage » incitant à l'action politique, y compris en l'absence d'un réseau de mobilisation préexistant à ce bouleversement affectif.

40.. Braud P., L'Emotion en politique. Problèmes d'analyse, Paris, Presses de Sciences Po., 1996, p. 181.

41.. En particulier le Special Courts Act, adopté peu après les événements de juin 1984, qui institua des tribunaux spéciaux, où l'identité des témoins pouvait demeurer secrète et dont les prévenus ne pouvaient contester les décisions devant la Haute Cour de la province, bien qu'une possibilité d'appel devant la Cour suprême leur soit reconnue. En 1985, une nouvelle loi vint institutionnaliser la terreur d'Etat au Pendjab : le Terrorism and Disruptive Activities (Prevention) Act, plus connu sous l'acronyme TADA. Ce texte 
reconnaît la validité d'aveux obtenus sous la torture et substitue la présomption de culpabilité à la présomption d'innocence. Le National Security Act (NSA) de 1987 autorise, quant à lui, la détention d'un individu sans procès pendant deux ans, cette période de détention pouvant être prolongée sur la base de nouvelles preuves de culpabilité apportées par l'accusation (c'est-à-dire par les services de l'Etat).

42.. Fenech L.E., Playing the Game of Love, op. cit., p. 41.

43.. Entretien, Chandigarh, 23 mars 2001.

44.. Ibid.

45.. Ouest du Penjab.

46.. Telford H., « The Political Economy of Punjab: Creating Space for Sikh Militancy », Asian Survey, 37(11), novembre 1992, pp. 969-987.

47.. Est du Penjab

48.. Bien que la place des femmes dans le mouvement armé pour le Khalistan soit souvent négligée, celles-ci apportèrent un soutien important à l'insurrection, soit de manière indirecte en servant de messagères aux groupes armés, soit de manière directe en s'engageant dans leurs rangs. Le nombre de femmes activement impliquées dans la lutte armée ne semble pourtant jamais avoir dépassé la dizaine, pour l'ensemble de la province.

49.. Le Breton D., Conduites à risques, Paris, PUF, 2002, p. 10, cité par Bozarslan H., « La figure du martyr chez les Kurdes ", Mayeur-Jaouen C., (dir.), Saints et héros du MoyenOrient contemporain, Paris, Maisonneuve et Larose, 2002, p. 347.

50.. Sur la place du « fun » dans certains mouvements nationalistes, dans une perspective anthropologique, voir Verkaaik O., Migrants and Militants. Fun and Urban Violence in Pakistan, Princeton, Princeton University Press, 2004.

51.. Schalk P., « Resistance and martyrdom in the process of state formation in Tamililam », Pettigrew J., (dir.), Martyrdom and Political Resistance, op. cit., p. 69 et suivantes.

52.. Entretien, Chandigarh, 23 mars 2001.

53.. Puri H.K., Judge P.S., Sekhon J.S., Terrorism in Punjab. Understanding Grassroots Reality, New Delhi, Har-Anand Publications, 1999, p. 68, tableau XII.

54.. Khosrokhavar F., « Le modèle Bassidji », Cultures \& Conflits, n²9-30, Paris, l'Harmattan, 1998, pp. 59-118.

55.. Blom A., « Les kamikazes du Cachemire, "martyrs" d'une cause perdue », Critique internationale, $\mathrm{n}^{\circ} 20$, juillet 2003 , p. 142 et suivantes.

56.. On ne peut s'empêcher de dresser ici un parallèle avec l'exaltation de la mort volontaire du stoïcisme, qui émergea à l'issue des guerres civiles de Rome, dans un contexte de fermeture de l'espace public, où « la liberté de mourir a remplacé la liberté d'agir ». Voir Pinguet M., La Mort volontaire au Japon, Paris, Gallimard, 1984, p. 15.

57.. Voir Keppley-Mahmood C., Fighting for Faith and Nation, op. cit.

58.. Khosrokhavar F., « La néo-umma transnationale : le martyr al-Qaeda », chap. 3, Les Nouveaux Martyrs d'Allah, Paris, Flammarion, 2002.

59.. Sur la dimension stratégique des attentats-suicides contemporains, voir Papp R., "The strategic logic of suicide terrorism », American Political Science Review, 97 (3), juillet 2003, pp. 20-32.

60.. Keppley-Mahmood C., Playing the Game of Love, op. cit., p. 29.

61.. Schalk P., « Resistance and martyrdom in the process of state formation in Tamililam », op. cit.

62.. Entretien, Londres, 22 juillet 1999. 
63. Pettigrew J., « Martyrdom and guerrilla organisation in Punjab », Journal of Commonwealth Politics, 30 (3), novembre 1992, p. 396.

64.. Entretien, Pendjab, 12 mars 2001.

65.. «O merveilleuse indépendance !"

66. Wah Nee Azadia, paroles et musique de Bhai Satnam Singh. Germany, chanté par Ranjit Singh Gillianwale, édité par le label Pamir Hakim, Allemagne, 1996. Nous remercions Ajit Singh Kera pour l'aide apportée dans la traduction.

67.. Keppley-Mahmood C., Playing the Game of Love, op. cit., p. 26.

68. Entre avril et octobre 1988, au moins deux cents militants de la Khalistan Commandor Force perdirent la vie, du fait de cette pratique et de l'infiltration de leur groupe, notamment de sa filière d'approvisionnement en armes ; Pettigrew J., «Martyrdom and guerrilla organisation in Punjab », op. cit., p. 395.

69.. Keppley-Mahmood C., Playing the Game of Love, op. cit., p. 29.

70.. Pettigrew J., The Sikhs of the Punjab, op. cit., p. 398.

71.. Voir Vinayak R., « Ominous intent », India Today, 15 mars 1999, pp. 34-35.

72.. C'est ce que révéla notamment l'interrogatoire d'un combattant khalistani chargé par l'ISI de préparer un attentat-suicide à Bombay, au moyen d'avions de tourisme : voir Raman B., "Suicide and suicidal terrorism », South Asia Analysis Group, Paper n 947, 12 mars 2004.

73.. Nous empruntons cette expression à Centlivres P., Centlivres-Demont M., « Les martyrs afghans par le texte et l'image (1978-1992) », Mayeur-Jaouen C., (dir.), Saints et héros du Moyen-Orient contemporain, op. cit., p. 330.

74.. Sluka J., « From graves to nations: political martyrdom and Irish nationalism », Pettigrew J., (dir.), Martyrdom and Political Resistance, op. cit., p. 39.

75.. Fenech L.E., Playing the Game of Love, op. cit., p. 45.

76.. Cité par Das V., « Time, self and community: features of the Sikh militant discourse ", Das V., Critical Events. An Anthropological Perspective on Contemporary India, New Delhi, Oxford University Press, 1995, p. 126.

77.. Pettigrew J., « Songs of the resistance movement », op. cit.

78. Bien que ces sites Internet soient aujourd'hui hors service, on peut retrouver une grande partie de ces clichés sur d'autres pages web, notamment à l'adresse suivante : http://www.searchsikhism.com/gal9.html ; on trouvera également les biographies des principaux martyrs du Khalistan sur http://www.neverforget84.com/1984.php.

79.. Fondateur de la BTFK.

80.. Fondateur du Babbar Khalsa International.

81.. Bien que cette page soit désormais inaccessible, le cliché est reproduit à l'adresse suivante : http://www.searchsikhism.com/images/khalistan/other/3/kulwinder.jpg. 82. Né dans le village de Budsinghwala (district de Faridkot) en 1967, celui-ci prit la tête de la Khalistan Liberation Force (KLF) à la mort de son fondateur, Avtar Singh Brama. 83.. On trouvera les mêmes clichés à l'adresse suivante : http:// allsikhkhalsa.tripod.com.

84.. Devji F., Landscapes of the Jihad. Militancy, Morality, Modernity, Delhi, Foundation Books, 2005, p. 95.

85.. Voir Gayer L., Les Politiques internationales de l'identité. Significations internationales des mobilisations identitaires des sikhs (Inde) et des Mohajirs (Pakistan), thèse de science politique, 2004, IEP de Paris, chap. 6, 6.1.4.1.4.

86.. On trouve en effet les mêmes mises en garde, pour accéder à ces clichés hyperviolents, que pour accéder aux sites pornographiques, comme le montre 
l'anthropologue Brian Axel, qui va jusqu'à parler de « pornotropisation de l'image du shahid », voir Axel B.K., The Nation's Tortured Body. Violence, Representation and the Formation of a Sikh Diaspora, Durham/Londres, Duke University Press, 2001, p. 144.

87.. Comme le note Louis E.Fenech, le martyrologe sikh, constitué par les récits ou les représentations picturales enthousiastes des martyrs sikhs, est profondément " idéaliste ", son but étant de convaincre les membres du Panth de la victoire ultime de la justice sur l'oppression, voir Fenech L.E., « The taunt in popular Sikh martyrologies ", Singh P., Barrier N.G., (dir.), The Transmission of Sikh Heritage in the Diaspora, Delhi, Manohar, 1996, p. 178.

88.. Bauman Z., Mortality, Immortality and Other Life Strategies, Oxford, Polity Press, 1992, pp. 26-27.

89.. Sur la fonction individualisante du martyre chez les militants d'Al-Qaïda, voir Devji F., Landscapes of the Jihad, op. cit., p. 120.

\section{RÉSUMÉS}

Si le Panth sikh a connu ses premiers martyrs dès le xviie siècle, ce n'est qu'au xixe que le mouvement de réforme socio-religieuse des Singh Sabhas a formalisé un idiome du martyre sikh, édictant les (bonnes) manières de pratiquer et d'évoquer la mort volontaire dans le sikhisme. Ces codes pragmatiques et discursifs ont été appropriés par les mouvements révolutionnaires sikhs (tels que le Ghadr, au début du xxe siècle) et surtout par le mouvement sécessionniste pour le Khalistan. Chez les Khalistanis, le martyre présente une profonde ambiguïté, puisque le désir de mort des combattants sikhs à longtemps constitué un obstacle à l'adoption d'une stratégie insurrectionnelle cohérente par les leaders des groupes armés. Lors de la commémoration de la lutte armée, en particulier sur l'Internet, ces suicides anomiques à peine voilés ont été reconstruits par les entrepreneurs identitaires sikhs comme des sacrifices offerts à la nation, dépouillés de leur dimension profondément individualiste.

Although the first Sikh martyrs gave their lives as early as the 17th century, it is only in the 19th that the Singh Sabha socio-religious reform movement standardized a Sikh "tradition" of martyrdom. This invented tradition was later on appropriated by Sikh revolutionary movements, such as the Ghadr movement of the 1910s, and even more so by the secessionist movement for Khalistan in the 1980s. For Khalistani guerillas, martyrdom was the outcome of a deeply individualistic death wish that prevented the leaders of the insurgent groups to define a rational strategy in their struggle against the Indian state. However, after the movement was militarily defeated, in the mid-1990s, these voluntary deaths were converted and celebrated (particularly on the Internet) as unambiguous sacrifices for the nation, and they were instrumental in the construction of a combative memory of the movement for Khalistan. 
INDEX

Index géographique : Inde

Mots-clés : guérillas et organisations clandestines, kamikaze (missions suicide), religion, séparatismes/ autonomismes, violence

\section{AUTEUR}

\section{LAURENT GAYER}

Laurent Gayer est docteur en sciences politiques (relations internationales) de l'IEP de Paris. Chercheur associé au Centre d'études de l'Inde et de l'Asie du Sud (CEIAS) depuis 2005, il dirige le programme « relations internationales » du Centre de sciences humaines (CSH) de New Delhi. Il prépare la publication de sa thèse : Les Politiques internationales de l'identité. Significations internationales des mobilisations identitaires des sikhs (Inde) et des Mohajirs (Pakistan). 